\title{
Exponential Stability of Impulsive Delayed Reaction-Diffusion Cellular Neural Networks via Poincaré Integral Inequality
}

\author{
Xianghong Lai and Tianxiang Yao \\ School of Economics \& Management, Nanjing University of Information Science \& Technology, Nanjing 210044, China \\ Correspondence should be addressed to Xianghong Lai; laixh1979@163.com
}

Received 15 November 2012; Accepted 8 February 2013

Academic Editor: Qi Luo

Copyright ( 2013 X. Lai and T. Yao. This is an open access article distributed under the Creative Commons Attribution License, which permits unrestricted use, distribution, and reproduction in any medium, provided the original work is properly cited.

\begin{abstract}
This work is devoted to the stability study of impulsive cellular neural networks with time-varying delays and reaction-diffusion terms. By means of new Poincaré integral inequality and Gronwall-Bellman-type impulsive integral inequality, we summarize some novel and concise sufficient conditions ensuring the global exponential stability of equilibrium point. The provided stability criteria are applicable to Dirichlet boundary condition and show that not only the reaction-diffusion coefficients but also the regional features including the boundary and dimension of spatial variable can influence the stability. Two examples are finally illustrated to demonstrate the effectiveness of our obtained results.
\end{abstract}

\section{Introduction}

Cellular neural networks (CNNs), proposed by Chua and Yang in $1988[1,2]$, have been the focus of a number of investigations due to their potential applications in various fields such as optimization, linear and nonlinear programming, associative memory, pattern recognition, and computer vision [3-7]. As the switching speed of neurons and amplifiers is finite in the implementation of neural networks, time delays are inevitable and therefore a type of more effective models is afterwards introduced, called delayed cellular neural networks (DCNNs). Actually, DCNNs have been found to be helpful in solving some dynamic image processing and pattern recognition problems.

As we all know, all the applications of CNNs and DCNNs depend heavily on the dynamic behaviors such as stability, convergence, and oscillatory $[8,9]$, wherein stability analysis is a major concern in the designs and applications. Correspondingly, the stability of CNNs and DCNNs is a subject of current interest and considerable theoretical efforts have been put into this topic with many good results reported (see, e.g., [10-13]).

With reference to neural networks, however, it is noteworthy that the state of electronic networks is often subject to instantaneous perturbations which may be caused by a switching phenomenon, frequency change, or other sudden noise. On this account, neural networks will experience abrupt change at certain instants, exhibiting impulse effects $[14,15]$. For instance, according to Arbib [16] and Haykin [17], when a stimulus from the body or the external environment is received by receptors, the electrical impulses will be conveyed to the neural net and impulse effects arise naturally in the net. In view of this discovery, many scientists have shown growing interests in the influence that the impulses may have on CNNs or DCNNs with a result that a large number of relevant results have been achieved (see, e.g., [18-24]).

Besides impulsive effects, diffusing effects are also nonignorable in reality since the diffusion is unavoidable when the electrons are moving in asymmetric electromagnetic fields. Therefore, the model of impulsive delayed reactiondiffusion neural networks appears as a natural description of the observed evolution phenomena of several real world problems. This one acknowledgement poses a new challenge to the stability research of neural networks.

So far, there have been some theoretical achievements [25-33] on the stability of impulsive delayed reactiondiffusion neural networks. Previously, authors of [27-32] studied the stability of impulsive delayed reaction-diffusion neural networks and put forward several stability criteria by impulsive differential inequality and Green formula, wherein 
the reaction-diffusion term is evaluated to be less than zero by means of Green formula and thereby the presented stability criteria are shown to be wholly independent of diffusion. According to this result, we fail to see the influence of diffusion on stability.

Recently, it is encouraging that, for impulsive delayed reaction-diffusion neural network, some new stability criteria involving diffusion are obtained in [25, 26, 33-36]. Meanwhile the estimation of reaction-diffusion term is not merely less than zero, instead a more accurate one is given; that is, the reaction-diffusion term is verified to be less than a negative definite term by using some inequalities together with Green formula. It is thereby testified that the diffusion does contribute to the stability of impulsive neural networks.

In [25], the authors quoted the following inequality to deal with the reaction-diffusion terms:

$$
\int_{\Omega^{*}}\left|\frac{\partial v(x)}{\partial x_{j}}\right|^{2} \mathrm{~d} x \geq \frac{1}{l_{j}^{2}} \int_{\Omega^{*}} v^{2}(x) \mathrm{d} x,
$$

where $\Omega^{*}$ is a cube $\left|x_{j}\right|<l_{j}(j=1,2, \ldots, m)$ and $v(x)$ is a real-valued function belonging to $C_{0}^{1}\left(\Omega^{*}\right)$. We can easily derive from this inequality that

$$
\int_{\Omega^{*}}|\nabla v|^{2} \mathrm{~d} x \geq\left(\int_{\Omega^{*}} v^{2}(x) \mathrm{d} x\right)\left(\sum_{j=1}^{m} \frac{1}{l_{j}^{2}}\right) .
$$

For better exploring the influence of diffusion on stability, we wonder if we can get a more accurate estimate of reaction-diffusion term. Fortunately, we find the following new Poincaré integral inequality supporting this idea:

$$
\int_{\mathcal{S}}|\nabla v(x)|^{2} \mathrm{~d} x \geq \frac{4 n}{B^{2}} \int_{\mathcal{S}} v^{2}(x) \mathrm{d} x .
$$

One can refer to Lemma 3 in Section 2 for the details of this inequality.

On the other hand, it is well known that the theory of differential and integral inequalities plays an important role in the qualitative and quantitative study of solution to differential equations. Up till now, there have been many applications of impulsive differential inequalities to impulsive dynamic systems, followed by lots of stability criteria provided. However, these stability criteria appear a bit complicated and we wonder if we can deduce relatively concise stability criteria by using impulsive integral inequalities

Motivated by these, we attempt to, for impulsive delayed neural networks, employ new Poincaré integral inequality to further investigate the influence of diffusion on the stability and combine Gronwall-Bellman-type impulsive integral inequality so as to provide some new and concise stability criteria. The rest of this paper is organized as follows. In Section 2, the model of impulsive cellular neural networks with time-varying delays and reaction-diffusion terms as well as Dirichlet boundary condition is outlined; in addition, some facts and lemmas are introduced for later reference. In Section 3, we provide a new estimate on the reactiondiffusion term by the agency of new Poincaré integral inequality and then discuss the global exponential stability of equilibrium point by utilizing Gronwall-Bellman-type impulsive integral inequality with a result of some novel and concise stability criteria presented. To conclude, two illustrative examples are given in Section 4 to verify the effectiveness of our obtained results.

\section{Preliminaries}

Let $R_{+}=[0, \infty)$ and $t_{0} \in R_{+}$. Let $R^{n}$ denote the $n$-dimensional Euclidean space, and let $\Omega=\prod_{i=1}^{m}\left[d_{i}, k_{i}\right]$ be a fixed rectangular region in $R^{m}$ and $M:=\max \left\{k_{i}-d_{i}: i=1, \ldots, m\right\}$. As usual, denote

$$
\begin{gathered}
C_{0}^{1}(\Omega)=\left\{v \mid v \text { and } D_{j} v=\frac{\partial v}{\partial x_{j}} \text { are continuous on } \Omega,\right. \\
\left.\left.v\right|_{\partial \Omega}=0,1 \leq j \leq m\right\} .
\end{gathered}
$$

Consider the following impulsive cellular neural network with time-varying delays and reaction-diffusion terms:

$$
\begin{gathered}
\frac{\partial u_{i}(t, x)}{\partial t}=\sum_{s=1}^{m} \frac{\partial}{\partial x_{s}}\left(D_{i s} \frac{\partial u_{i}(t, x)}{\partial x_{s}}\right)-a_{i} u_{i}(t, x) \\
+\sum_{j=1}^{n} b_{i j} f_{j}\left(u_{j}(t, x)\right)+\sum_{j=1}^{n} c_{i j} f_{j}\left(u_{j}\left(t-\tau_{j}(t), x\right)\right) \\
t \geq t_{0}, t \neq t_{k}, x \in \Omega \\
i=1,2, \ldots, n, k=1,2, \ldots \\
u_{i}\left(t_{k}+0, x\right)=u_{i}\left(t_{k}, x\right)+P_{i k}\left(u_{i}\left(t_{k}, x\right)\right) \\
x \in \Omega, i=1,2, \ldots, n, k=1,2, \ldots,
\end{gathered}
$$

where $n$ corresponds to the numbers of units in a neural network; $x=\left(x_{1}, \ldots, x_{m}\right)^{\mathrm{T}} \in \Omega, u_{i}(t, x)$ denotes the state of the $i$ th neuron at time $t$ and in space $x ; D_{i s}=$ const $>0$ represents transmission diffusion of the $i$ th unit; activation function $f_{j}\left(u_{j}(t, x)\right)$ stands for the output of the $j$ th unit at time $t$ and in space $x ; b_{i j}, c_{i j}$, and $a_{i}$ are constants: $b_{i j}$ indicates the connection strength of the $j$ th unit on the $i$ th unit at time $t$ and in space $x, c_{i j}$ denotes the connection weight of the $j$ th unit on the $i$ th unit at time $t-\tau_{j}(t)$ and in space $x$, where $\tau_{j}(t)$ corresponds to the transmission delay along the axon of the $j$ th unit, satisfying $0 \leq \tau_{j}(t) \leq \tau(\tau=$ const $)$ and $\dot{\tau}_{j}(t)<(1-$ $(1 / h))(h>0)$, and $a_{i}>0$ represents the rate with which the $i$ th unit will reset its potential to the resting state in isolation when disconnected from the network and external inputs at time $t$ and in space $x$. The fixed moments $t_{k}(k=1,2, \ldots)$ are called impulsive moments meeting $0 \leq t_{0}<t_{1}<t_{2}<\cdots$ and $\lim _{k \rightarrow \infty} t_{k}=\infty ; u_{i}\left(t_{k}+0, x\right)$ and $u_{i}\left(t_{k}-0, x\right)$ represent the right-hand and left-hand limit of $u_{i}(t, x)$ at time $t_{k}$ and in space $x$, respectively. $P_{i k}\left(u_{i}\left(t_{k}, x\right)\right)$ stands for the abrupt change of $u_{i}(t, x)$ at the impulsive moment $t_{k}$ and in space $x$. 
Denote by $u(t, x)=u\left(t, x ; t_{0}, \varphi\right), u \in R^{n}$, the solution of system (5)-(6), satisfying the initial condition

$$
u\left(s, x ; t_{0}, \varphi\right)=\varphi(s, x), \quad t_{0}-\tau \leq s \leq t_{0}, x \in \Omega,
$$

and Dirichlet boundary condition

$$
u\left(t, x ; t_{0}, \varphi\right)=0, \quad t \geq t_{0}, x \in \partial \Omega,
$$

where the vector-valued function $\varphi(s, x)=\left(\varphi_{1}(s, x), \ldots\right.$, $\left.\varphi_{n}(s, x)\right)^{\mathrm{T}}$ is such that $\int_{\Omega} \sum_{i=1}^{n} \varphi_{i}^{2}(s, x) \mathrm{d} x$ is bounded on $\left[t_{0}-\right.$ $\left.\tau, t_{0}\right]$.

The solution $u(t, x)=u\left(t, x ; t_{0}, \varphi\right)=\left(u_{1}\left(t, x ; t_{0}, \varphi\right), \ldots\right.$, $\left.u_{n}\left(t, x ; t_{0}, \varphi\right)\right)^{\mathrm{T}}$ of problem (5)-(8) is, for the time variable $t, \mathrm{a}$ piecewise continuous function with the first kind discontinuity at the points $t_{k}(k=1,2, \ldots)$, where it is continuous from the left; that is, the following relations are true:

$$
\begin{gathered}
u_{i}\left(t_{k}-0, x\right)=u_{i}\left(t_{k}, x\right), \\
u_{i}\left(t_{k}+0, x\right)=u_{i}\left(t_{k}, x\right)+P_{i k}\left(u_{i}\left(t_{k}, x\right)\right) .
\end{gathered}
$$
by

Throughout this paper, the norm of $u\left(t, x ; t_{0}, \varphi\right)$ is defined

$$
\left\|u\left(t, x ; t_{0}, \varphi\right)\right\|_{\Omega}^{2}=\sum_{i=1}^{n} \int_{\Omega} u_{i}^{2}\left(t, x ; t_{0}, \varphi\right) \mathrm{d} x .
$$
lows:

Before proceeding, we introduce two hypotheses as fol-

(H1) $f_{i}(\bullet): R \rightarrow R$ satisfies $f_{i}(0)=0$, and there exists a constant $l_{i}>0$ such that $\left|f_{i}\left(y_{1}\right)-f_{i}\left(y_{2}\right)\right| \leq l_{i}\left|y_{1}-y_{2}\right|$ for all $y_{1}, y_{2} \in R$ and $i=1,2, \ldots, n$.

$(\mathrm{H} 2) P_{i k}(\bullet): R \rightarrow R$ is continuous and $P_{i k}(0)=0, i=$ $1,2, \ldots, n, k=1,2, \ldots$

According to (H1) and (H2), it is easy to see that problem (5)-(8) admits an equilibrium point $u=0$.

Definition 1 (see [25]). The equilibrium point $u=0$ of problem (5)-(8) is said to be globally exponentially stable if there exist constants $\kappa>0$ and $\omega \geq 1$ such that

$$
\left\|u\left(t, x ; t_{0}, \varphi\right)\right\|_{\Omega} \leq \omega \overline{\|\varphi\|_{\Omega}} \mathrm{e}^{-\kappa\left(t-t_{0}\right)}, \quad t \geq t_{0},
$$

where ${\overline{\|\varphi\|_{\Omega}}}_{\Omega}^{2}=\sup _{t_{0}-\tau \leq s \leq t_{0}} \sum_{i=1}^{n} \int_{\Omega} \varphi_{i}^{2}(s, x) \mathrm{d} x$.

Lemma 2 (see [37] Gronwall-Bellman-type Impulsive Integral Inequality). Assume that

(A1) the sequence $\left\{t_{k}\right\}$ satisfies $0 \leq t_{0}<t_{1}<t_{2}<\cdots$, with $\lim _{k \rightarrow \infty} t_{k}=\infty$,

(A2) $q \in P C^{1}\left[R_{+}, R\right]$ and $q(t)$ is left-continuous at $t_{k}, k=$ $1,2, \ldots$,

(A3) $p \in C\left[R_{+}, R_{+}\right]$and for $k=1,2, \ldots$,

$$
q(t) \leq c+\int_{t_{0}}^{t} p(s) q(s) \mathrm{d} s+\sum_{t_{0}<t_{k}<t} \eta_{k} q\left(t_{k}\right), \quad t \geq t_{0},
$$

where $\eta_{k} \geq 0$ and $c=$ const. Then,

$$
q(t) \leq c \prod_{t_{0}<t_{k}<t}\left(1+\eta_{k}\right) \exp \left(\int_{t_{0}}^{t} p(s) \mathrm{d} s\right), \quad t \geq t_{0} .
$$

Lemma 3 (see [38] Poincaré integral inequality). Let $\mathcal{S}=$ $\prod_{i=1}^{n}\left[a_{i}, b_{i}\right]$ be a fixed rectangular region in $R^{n}$ and $B:=$ $\max \left\{b_{i}-a_{i}: i=1, \ldots, n\right\}$. For any $v(x) \in C_{0}^{1}(\mathcal{S})$,

$$
\int_{\mathcal{S}} v^{2}(x) \mathrm{d} x \leq \frac{B^{2}}{4 n} \int_{\mathcal{S}}|\nabla v(x)|^{2} \mathrm{~d} x .
$$

Remark 4. According to Lemma 2.1 in [25], we know if $\mathcal{S}$ is a cube $\left|x_{j}\right|<l_{j}(j=1,2, \ldots, m)$ and $v(x)$ is a real-valued function belonging to $C_{0}^{1}(\delta)$, then

$$
\int_{\mathcal{S}}\left|\frac{\partial v(x)}{\partial x_{j}}\right|^{2} \mathrm{~d} x \geq \frac{1}{l_{j}^{2}} \int_{\mathcal{S}} v^{2}(x) \mathrm{d} x
$$

which yields

$$
\int_{\mathcal{S}}|\nabla v|^{2} \mathrm{~d} x \geq\left(\int_{\mathcal{S}} v^{2}(x) \mathrm{d} x\right)\left(\sum_{j=1}^{m} \frac{1}{l_{j}^{2}}\right)
$$

Through the simple example as follows, we can find that in some cases the estimate $\int_{\mathcal{S}}|\nabla v(x)|^{2} \mathrm{~d} x \geq\left(4 n / B^{2}\right) \int_{\mathcal{S}} v^{2}(x) \mathrm{d} x$ shown in Lemma 3 can do better. Let $\mathcal{S}=[0,1] \times[0,2]$, we derive from Lemma 2.1 in [25] that

$$
\int_{\mathcal{S}}|\nabla v|^{2} \mathrm{~d} x \geq\left(\int_{\mathcal{S}} v^{2}(x) \mathrm{d} x\right)\left(\sum_{j=1}^{m} \frac{1}{l_{j}^{2}}\right)=\frac{5}{4} \int_{\mathcal{S}} v^{2}(x) \mathrm{d} x
$$

whereas the application of Lemma 3 of this paper will give

$$
\int_{\mathcal{S}}|\nabla v(x)|^{2} \mathrm{~d} x \geq \frac{4 n}{B^{2}} \int_{\mathcal{S}} v^{2}(x) \mathrm{d} x=2 \int_{\mathcal{S}} v^{2}(x) \mathrm{d} x,
$$

which is obviously superior to $\int_{\mathcal{S}}|\nabla v|^{2} \mathrm{~d} x \geq$ $\left(\int_{\mathcal{S}} v^{2}(x) \mathrm{d} x\right)$.

\section{Main Results}

Theorem 5. Provided that one has the following:

(1) let $\underline{D}=\min \left\{D_{i s}: i=1, \ldots, n ; s=1, \ldots, m\right\}>0$ and denote $8 m \underline{D} / M^{2}=\chi$;

(2) $P_{i k}\left(u_{i}\left(t_{k}, x\right)\right)=-\theta_{i k} u_{i}\left(t_{k}, x\right), 0 \leq \theta_{i k} \leq 2$;

(3) there exists a constant $\gamma>0$ satisfying $\gamma+\lambda+h \rho e^{\gamma \tau}>0$ as well as $\lambda+h \rho e^{\gamma \tau}<0$, where $\lambda=\max _{i=1, \ldots, n}(-\chi-$ $\left.2 a_{i}+\sum_{j=1}^{n}\left(b_{i j}^{2}+c_{i j}^{2}\right)\right)+\rho, \rho=n \max _{i=1, \ldots, n}\left(l_{i}^{2}\right) ;$

then, the equilibrium point $u=0$ of problem (5)-(8) is globally exponentially stable with convergence rate $-\left(\lambda+h \rho e^{\gamma \tau}\right) / 2$. 
Proof. Multiplying both sides of $(5)$ by $u_{i}(t, x)$ and integrating with respect to spatial variable $x$ on $\Omega$, we get

$$
\begin{aligned}
& \frac{\mathrm{d}\left(\int_{\Omega} u_{i}^{2}(t, x) \mathrm{d} x\right)}{\mathrm{d} t} \\
& =2 \sum_{s=1}^{m} \int_{\Omega} u_{i}(t, x) \frac{\partial}{\partial x_{s}}\left(D_{i s} \frac{\partial u_{i}(t, x)}{\partial x_{s}}\right) \mathrm{d} x \\
& \quad-2 a_{i} \int_{\Omega} u_{i}^{2}(t, x) \mathrm{d} x \\
& +2 \sum_{j=1}^{n} b_{i j} \int_{\Omega} u_{i}(t, x) f_{j}\left(u_{j}(t, x)\right) \mathrm{d} x \\
& +2 \sum_{j=1}^{n} c_{i j} \int_{\Omega} u_{i}(t, x) f_{j}\left(u_{j}\left(t-\tau_{j}(t), x\right)\right) \mathrm{d} x \\
& t \geq t_{0}, t \neq t_{k}, i=1, \ldots, n, k=1,2, \ldots
\end{aligned}
$$

Regarding the right-hand part of (19), the first term becomes by using Green formula, Dirichlet boundary condition, Lemma 3, and condition (1) of Theorem 5

$$
\begin{aligned}
& 2 \sum_{s=1}^{m} \int_{\Omega} u_{i}(t, x) \frac{\partial}{\partial x_{s}}\left(D_{i s} \frac{\partial u_{i}(t, x)}{\partial x_{s}}\right) \mathrm{d} x \\
& \quad=-2 \sum_{s=1}^{m} \int_{\Omega} D_{i s}\left(\frac{\partial u_{i}(t, x)}{\partial x_{s}}\right)^{2} \mathrm{~d} x \\
& \quad \leq \frac{-8 m \underline{D}}{M^{2}} \int_{\Omega} u_{i}^{2}(t, x) \mathrm{d} x \triangleq-\chi \int_{\Omega} u_{i}^{2}(t, x) \mathrm{d} x .
\end{aligned}
$$

Moreover, From (H1), we have

$$
\begin{aligned}
& 2 \sum_{j=1}^{n} b_{i j} \int_{\Omega} u_{i}(t, x) f_{j}\left(u_{j}(t, x)\right) \mathrm{d} x \\
& \quad \leq 2 \sum_{j=1}^{n}\left|b_{i j}\right| \int_{\Omega}\left|u_{i}(t, x)\right|\left|f_{j}\left(u_{j}(t, x)\right)\right| \mathrm{d} x \\
& \quad \leq 2 \sum_{j=1}^{n} \int_{\Omega} l_{j}\left|b_{i j}\right|\left|u_{i}(t, x)\right|\left|u_{j}(t, x)\right| \mathrm{d} x \\
& \quad \leq \sum_{j=1}^{n} \int_{\Omega}\left(b_{i j}^{2} u_{i}^{2}(t, x)+l_{j}^{2} u_{j}^{2}(t, x)\right) \mathrm{d} x, \\
& 2 \sum_{j=1}^{n} c_{i j} \int_{\Omega} u_{i}(t, x) f_{j}\left(u_{j}\left(t-\tau_{j}(t), x\right)\right) \mathrm{d} x \\
& \quad \leq 2 \sum_{j=1}^{n}\left|c_{i j}\right| \int_{\Omega}\left|u_{i}(t, x)\right|\left|f_{j}\left(u_{j}\left(t-\tau_{j}(t), x\right)\right)\right| \mathrm{d} x \\
& \quad \leq 2 \sum_{j=1}^{n} \int_{\Omega} l_{j}\left|c_{i j}\right|\left|u_{i}(t, x)\right|\left|u_{j}\left(t-\tau_{j}(t), x\right)\right| \mathrm{d} x \\
& \quad \sum_{j=1}^{n} \int_{\Omega}\left(c_{i j}^{2} u_{i}^{2}(t, x)+l_{j}^{2} u_{j}^{2}\left(t-\tau_{j}(t), x\right)\right) \mathrm{d} x . \\
& \quad
\end{aligned}
$$

Consequently, substituting (20)-(22) into (19) produces

$$
\begin{aligned}
& \frac{\mathrm{d}\left(\int_{\Omega} u_{i}^{2}(t, x) \mathrm{d} x\right)}{\mathrm{d} t} \\
& \leq-\chi \int_{\Omega} u_{i}^{2}(t, x) \mathrm{d} x-2 a_{i} \int_{\Omega} u_{i}^{2}(t, x) \mathrm{d} x \\
& \quad+\sum_{j=1}^{n} \int_{\Omega}\left(b_{i j}^{2} u_{i}^{2}(t, x)+l_{j}^{2} u_{j}^{2}(t, x)\right) \mathrm{d} x \\
& \quad+\sum_{j=1}^{n} \int_{\Omega}\left(c_{i j}^{2} u_{i}^{2}(t, x)+l_{j}^{2} u_{j}^{2}\left(t-\tau_{j}(t), x\right)\right) \mathrm{d} x
\end{aligned}
$$

for $t \geq t_{0}, t \neq t_{k}, i=1, \ldots, n, k=1,2, \ldots$.

Define a Lyapunov function $V_{i}(t)$ as $V_{i}(t)=\int_{\Omega} u_{i}^{2}(t, x) \mathrm{d} x$. It is easy to find that $V_{i}(t)$ is a piecewise continuous function with the first kind discontinuous points $t_{k}(k=1,2, \ldots)$, where it is continuous from the left, that is, $V_{i}\left(t_{k}-0\right)=$ $V_{i}\left(t_{k}\right)(k=1,2, \ldots)$. In addition, we also see

$$
V_{i}\left(t_{k}+0\right) \leq V_{i}\left(t_{k}\right), \quad k=0,1,2, \ldots
$$

as $V_{i}\left(t_{0}+0\right) \leq V_{i}\left(t_{0}\right)$ and the following estimate derived from condition (2) of Theorem 5:

$$
\begin{array}{r}
u_{i}^{2}\left(t_{k}+0, x\right)=\left(-\theta_{i k} u_{i}\left(t_{k}, x\right)+u_{i}\left(t_{k}, x\right)\right)^{2} \\
=\left(1-\theta_{i k}\right)^{2} u_{i}^{2}\left(t_{k}, x\right) \leq u_{i}^{2}\left(t_{k}, x\right), \\
k=1,2, \ldots .
\end{array}
$$

Put $t \in\left(t_{k}, t_{k+1}\right), k=0,1,2, \ldots$. It then results from (23) that

$$
\begin{aligned}
\frac{\mathrm{d} V_{i}(t)}{\mathrm{d} t} \leq & -\chi \int_{\Omega} u_{i}^{2}(t, x) \mathrm{d} x-2 a_{i} \int_{\Omega} u_{i}^{2}(t, x) \mathrm{d} x \\
& +\sum_{j=1}^{n} \int_{\Omega}\left(b_{i j}^{2} u_{i}^{2}(t, x)+l_{j}^{2} u_{j}^{2}(t, x)\right) \mathrm{d} x \\
& +\sum_{j=1}^{n} \int_{\Omega}\left(c_{i j}^{2} u_{i}^{2}(t, x)+l_{j}^{2} u_{j}^{2}\left(t-\tau_{j}(t), x\right)\right) \mathrm{d} x \\
\leq & \left(-\chi-2 a_{i}+\sum_{j=1}^{n} b_{i j}^{2}+\sum_{j=1}^{n} c_{i j}^{2}\right) V_{i}(t) \\
& +\max _{i=1, \ldots, n}\left(l_{i}^{2}\right) \sum_{j=1}^{n} V_{j}(t) \\
& +\max _{i=1, \ldots, n}\left(l_{i}^{2}\right) \sum_{j=1}^{n} V_{j}\left(t-\tau_{j}(t)\right) \\
& t \in\left(t_{k}, t_{k+1}\right), k=0,1,2, \cdots .
\end{aligned}
$$



reads

Choose $V(t)$ of the form $V(t)=\sum_{i=1}^{n} V_{i}(t)$. From (26), one

$$
\begin{array}{r}
\frac{\mathrm{d} V(t)}{\mathrm{d} t} \leq \lambda V(t)+\rho \sum_{j=1}^{n} V_{j}\left(t-\tau_{j}(t)\right), \\
t \in\left(t_{k}, t_{k+1}\right), k=0,1,2, \ldots,
\end{array}
$$

where $\lambda=\max _{i=1, \ldots, n}\left(-\chi-2 a_{i}+\sum_{j=1}^{n}\left(b_{i j}^{2}+c_{i j}^{2}\right)\right)+\rho$ and $\rho=$ $n \max _{i=1, \ldots, n}\left(l_{i}^{2}\right)$.

Now construct $V^{*}(t)=\mathrm{e}^{\gamma\left(t-t_{0}\right)} V(t)$ again, where $\gamma>0$ satisfies $\gamma+\lambda+h \rho \mathrm{e}^{\gamma \tau}>0$ and $\lambda+h \rho \mathrm{e}^{\gamma \tau}<0$. Evidently, $V^{*}(t)$ is also a piecewise continuous function with the first kind discontinuous points $t_{k}(k=1,2, \ldots)$, where it is continuous from the left, that is, $V^{*}\left(t_{k}-0\right)=V^{*}\left(t_{k}\right)(k=1,2, \ldots)$. Moreover, at $t=t_{k}(k=0,1,2, \ldots)$, we find by use of (24)

$$
V^{*}\left(t_{k}+0\right) \leq V^{*}\left(t_{k}\right), \quad k=0,1,2, \ldots
$$

Set $t \in\left(t_{k}, t_{k+1}\right), k=0,1,2, \ldots$ By virtue of (27), one has

$$
\begin{array}{rl}
\frac{\mathrm{d} V^{*}(t)}{\mathrm{d} t}= & \gamma \mathrm{e}^{\gamma\left(t-t_{0}\right)} V(t)+\mathrm{e}^{\gamma\left(t-t_{0}\right)} \frac{\mathrm{d} V(t)}{\mathrm{d} t} \\
\leq & \gamma \mathrm{e}^{\gamma\left(t-t_{0}\right)} V(t) \\
& +\left(\lambda V(t)+\rho \sum_{j=1}^{n} V_{j}\left(t-\tau_{j}(t)\right)\right) \mathrm{e}^{\gamma\left(t-t_{0}\right)} \\
= & (\gamma+\lambda) V^{*}(t)+\rho \mathrm{e}^{\gamma\left(t-t_{0}\right)} \sum_{j=1}^{n} V_{j}\left(t-\tau_{j}(t)\right) \\
t & t \in\left(t_{k}, t_{k+1}\right), k=0,1,2, \ldots
\end{array}
$$

Choose small enough $\varepsilon>0$. Integrating (29) from $t_{k}+\varepsilon$ to $t$ gives

$$
\begin{array}{r}
V^{*}(t) \leq V^{*}\left(t_{k}+\varepsilon\right)+(\gamma+\lambda) \int_{t_{k}+\varepsilon}^{t} V^{*}(s) \mathrm{d} s \\
+\int_{t_{k}+\varepsilon}^{t} \rho \mathrm{e}^{\gamma\left(s-t_{0}\right)} \sum_{j=1}^{n} V_{j}\left(s-\tau_{j}(s)\right) \mathrm{d} s \\
t \in\left(t_{k}, t_{k+1}\right), \quad k=0,1,2, \ldots,
\end{array}
$$

which yields, after letting $\varepsilon \rightarrow 0$ in (30),

$$
\begin{array}{r}
V^{*}(t) \leq V^{*}\left(t_{k}+0\right)+(\gamma+\lambda) \int_{t_{k}}^{t} V^{*}(s) \mathrm{d} s \\
+\int_{t_{k}}^{t} \rho \mathrm{e}^{\gamma\left(s-t_{0}\right)} \sum_{j=1}^{n} V_{j}\left(s-\tau_{j}(s)\right) \mathrm{d} s \\
t \in\left(t_{k}, t_{k+1}\right), k=0,1,2, \ldots
\end{array}
$$

Next we will estimate the value of $V^{*}(t)$ at $t=t_{k+1}, k=$ $0,1,2, \ldots$. For small enough $\varepsilon>0$, we put $t=t_{k+1}-\varepsilon$. An application of (31) leads to, for $k=0,1,2, \ldots$,

$$
\begin{aligned}
V^{*}\left(t_{k+1}-\varepsilon\right) \leq & V^{*}\left(t_{k}+0\right)+(\gamma+\lambda) \int_{t_{k}}^{t_{k+1}-\varepsilon} V^{*}(s) \mathrm{d} s \\
& +\int_{t_{k}}^{t_{k+1}-\varepsilon} \rho \mathrm{e}^{\gamma\left(s-t_{0}\right)} \sum_{j=1}^{n} V_{j}\left(s-\tau_{j}(s)\right) \mathrm{d} s .
\end{aligned}
$$

If we let $\varepsilon \rightarrow 0$ in (32), there results

$$
\begin{array}{r}
V^{*}\left(t_{k+1}-0\right) \leq V^{*}\left(t_{k}+0\right)+(\gamma+\lambda) \int_{t_{k}}^{t_{k+1}} V^{*}(s) \mathrm{d} s \\
+\int_{t_{k}}^{t_{k+1}} \rho \mathrm{e}^{\gamma\left(s-t_{0}\right)} \sum_{j=1}^{n} V_{j}\left(s-\tau_{j}(s)\right) \mathrm{d} s \\
k=0,1,2, \ldots
\end{array}
$$

Note that $V^{*}\left(t_{k+1}-0\right)=V^{*}\left(t_{k+1}\right)$ is applicable for $k=$ $0,1,2, \ldots$ Thus,

$$
\begin{aligned}
V^{*}\left(t_{k+1}\right) \leq & V^{*}\left(t_{k}+0\right)+(\gamma+\lambda) \int_{t_{k}}^{t_{k+1}} V^{*}(s) \mathrm{d} s \\
& +\int_{t_{k}}^{t_{k+1}} \rho \mathrm{e}^{\gamma\left(s-t_{0}\right)} \sum_{j=1}^{n} V_{j}\left(s-\tau_{j}(s)\right) \mathrm{d} s
\end{aligned}
$$

holds for $k=0,1,2, \ldots$ By synthesizing (31) and (34), we then arrive at

$$
\begin{array}{r}
V^{*}(t) \leq V^{*}\left(t_{k}+0\right)+(\gamma+\lambda) \int_{t_{k}}^{t} V^{*}(s) \mathrm{d} s \\
+\int_{t_{k}}^{t} \rho \mathrm{e}^{\gamma\left(s-t_{0}\right)} \sum_{j=1}^{n} V_{j}\left(s-\tau_{j}(s)\right) \mathrm{d} s \\
t \in\left(t_{k}, t_{k+1}\right], \quad k=0,1,2, \ldots
\end{array}
$$

This, together with (28), results in

$$
\begin{aligned}
V^{*}(t) \leq & V^{*}\left(t_{k}\right)+(\gamma+\lambda) \int_{t_{k}}^{t} V^{*}(s) \mathrm{d} s \\
& +\int_{t_{k}}^{t} \rho \mathrm{e}^{\gamma\left(s-t_{0}\right)} \sum_{j=1}^{n} V_{j}\left(s-\tau_{j}(s)\right) \mathrm{d} s
\end{aligned}
$$

for $t \in\left(t_{k}, t_{k+1}\right], k=0,1,2, \ldots$.

Recalling assumptions that $0 \leq \tau_{j}(t) \leq \tau$ and $\dot{\tau}_{j}(t)<$ $(1-(1 / h))(h>0)$, we obtain

$$
\begin{aligned}
& \int_{t_{k}}^{t} \rho \mathrm{e}^{\gamma\left(s-t_{0}\right)} \sum_{j=1}^{n} V_{j}\left(s-\tau_{j}(s)\right) \mathrm{d} s \\
& \quad=\sum_{j=1}^{n} \int_{t_{k}-\tau_{j}\left(t_{k}\right)}^{t-\tau_{j}(t)} \rho \mathrm{e}^{\gamma\left(\theta+\tau_{j}(s)-t_{0}\right)} V_{j}(\theta) \frac{1}{1-\dot{\tau}_{j}(s)} \mathrm{d} \theta \\
& \quad \leq h \rho \mathrm{e}^{\gamma \tau} \sum_{j=1}^{n} \int_{t_{k}-\tau_{j}\left(t_{k}\right)}^{t-\tau_{j}(t)} \mathrm{e}^{\gamma\left(\theta-t_{0}\right)} V_{j}(\theta) \mathrm{d} \theta .
\end{aligned}
$$


Hence,

$$
\begin{array}{r}
V^{*}(t) \leq V^{*}\left(t_{k}\right)+(\gamma+\lambda) \int_{t_{k}}^{t} V^{*}(s) \mathrm{d} s \\
+h \rho \mathrm{e}^{\gamma \tau} \sum_{j=1}^{n} \int_{t_{k}-\tau_{j}\left(t_{k}\right)}^{t-\tau_{j}(t)} \mathrm{e}^{\gamma\left(s-t_{0}\right)} V_{j}(s) \mathrm{d} s \\
t \in\left(t_{k}, t_{k+1}\right], \quad k=0,1,2, \ldots
\end{array}
$$

By induction argument, we reach

$$
\begin{aligned}
& V^{*}\left(t_{k}\right) \leq V^{*}\left(t_{k-1}\right)+(\gamma+\lambda) \int_{t_{k-1}}^{t_{k}} V^{*}(s) \mathrm{d} s \\
&+h \rho \mathrm{e}^{\gamma \tau} \sum_{j=1}^{n} \int_{t_{k-1}-\tau_{j}\left(t_{k-1}\right)}^{t_{k}-\tau_{j}\left(t_{k}\right)} \mathrm{e}^{\gamma\left(s-t_{0}\right)} V_{j}(s) \mathrm{d} s, \\
& \vdots \\
& V^{*}\left(t_{2}\right) \leq V^{*}\left(t_{1}\right)+(\gamma+\lambda) \int_{t_{1}}^{t_{2}} V^{*}(s) \mathrm{d} s \\
&+h \rho \mathrm{e}^{\gamma \tau} \sum_{j=1}^{n} \int_{t_{1}-\tau_{j}\left(t_{1}\right)}^{t_{2}-\tau_{j}\left(t_{2}\right)} \mathrm{e}^{\gamma\left(s-t_{0}\right)} V_{j}(s) \mathrm{d} s, \\
& V^{*}\left(t_{1}\right) \leq V^{*}\left(t_{0}\right)+(\gamma+\lambda) \int_{t_{0}}^{t_{1}} V^{*}(s) \mathrm{d} s \\
&+h \rho \mathrm{e}^{\gamma \tau} \sum_{j=1}^{n} \int_{t_{0}-\tau_{j}\left(t_{0}\right)}^{t_{1}-\tau_{j}\left(t_{1}\right)} \mathrm{e}^{\gamma\left(s-t_{0}\right)} V_{j}(s) \mathrm{d} s .
\end{aligned}
$$

Therefore,

$$
\begin{aligned}
V^{*}(t) \leq & V^{*}\left(t_{0}\right)+(\gamma+\lambda) \int_{t_{0}}^{t} V^{*}(s) \mathrm{d} s \\
& +h \rho \mathrm{e}^{\gamma \tau} \sum_{j=1}^{n} \int_{t_{0}-\tau_{j}\left(t_{0}\right)}^{t-\tau_{j}(t)} \mathrm{e}^{\gamma\left(s-t_{0}\right)} V_{j}(s) \mathrm{d} s \\
\leq & V^{*}\left(t_{0}\right)+(\gamma+\lambda) \int_{t_{0}}^{t} V^{*}(s) \mathrm{d} s \\
& +h \rho \mathrm{e}^{\gamma \tau} \sum_{j=1}^{n} \int_{t_{0}-\tau_{j}\left(t_{0}\right)}^{t} \mathrm{e}^{\gamma\left(s-t_{0}\right)} V_{j}(s) \mathrm{d} s \\
= & V^{*}\left(t_{0}\right)+\left(\gamma+\lambda+h \rho \mathrm{e}^{\gamma \tau}\right) \int_{t_{0}}^{t} V^{*}(s) \mathrm{d} s \\
& +h \rho \mathrm{e}^{\gamma \tau} \sum_{j=1}^{n} \int_{t_{0}-\tau_{j}\left(t_{0}\right)}^{t_{0}} \mathrm{e}^{\gamma\left(s-t_{0}\right)} V_{j}(s) \mathrm{d} s \\
& t \in\left(t_{k}, t_{k+1}\right], k=0,1,2, \ldots
\end{aligned}
$$

Since

$$
\begin{aligned}
& h \rho \mathrm{e}^{\gamma \tau} \sum_{j=1}^{n} \int_{t_{0}-\tau_{j}\left(t_{0}\right)}^{t_{0}} \mathrm{e}^{\gamma\left(s-t_{0}\right)} V_{j}(s) \mathrm{d} s \\
& \leq h \rho \mathrm{e}^{\gamma \tau} \sum_{j=1}^{n} \int_{t_{0}-\tau}^{t_{0}} V_{j}(s) \mathrm{d} s \\
& =h \rho \mathrm{e}^{\gamma \tau} \int_{t_{0}-\tau}^{t_{0}}\left(\sum_{j=1}^{n} \int_{\Omega} \varphi_{j}^{2}(s, x) \mathrm{d} x\right) \mathrm{d} s \\
& \leq \tau h \rho \mathrm{e}^{\gamma \tau} \overline{\|\varphi\|_{\Omega}^{2}},
\end{aligned}
$$

we claim

$$
\begin{aligned}
V^{*}(t) \leq & V^{*}\left(t_{0}\right)+\tau h \rho \mathrm{e}^{\gamma \tau}\|\varphi\|_{\Omega}^{2} \\
+ & \left(\gamma+\lambda+h \rho \mathrm{e}^{\gamma \tau}\right) \int_{t_{0}}^{t} V^{*}(s) \mathrm{d} s \\
& t \in\left(t_{k}, t_{k+1}\right], k=0,1,2 \ldots
\end{aligned}
$$

According to Lemma 2, we know

$$
\begin{aligned}
V^{*}(t) \leq & \left(V^{*}\left(t_{0}\right)+\tau h \rho \mathrm{e}^{\gamma \tau}{\overline{\|\varphi\|_{\Omega}^{2}}}^{2}\right. \\
& \times \exp \left\{\left(\gamma+\lambda+h \rho \mathrm{e}^{\gamma \tau}\right)\left(t-t_{0}\right)\right\}, \quad t \geq t_{0}
\end{aligned}
$$

which reduces to

$$
\begin{array}{r}
\left\|u\left(t, x ; t_{0}, \varphi\right)\right\|_{\Omega} \\
\leq \sqrt{1+\tau h \rho \mathrm{e}^{\gamma \tau}} \overline{\|\varphi\|_{\Omega}} \exp \left\{\left(\frac{\lambda+h \rho \mathrm{e}^{\gamma \tau}}{2}\right)\left(t-t_{0}\right)\right\}, \\
t \geq t_{0} .
\end{array}
$$

This completes the proof.

Remark 6. According to Theorem 5, we see that the diffusion can really influence the stability of equilibrium point $u=0$ of problem (5)-(8), wherein the factors embrace not only the reaction-diffusion coefficients but also the regional features including the dimension and boundary of spatial variable. Owing to the employ of new Poincaré integral inequality, in this paper, the estimation of reaction-diffusion terms is superior to that in [25] in some cases, and this will be helpful to further know the influence of diffusion on stability. What is more, from condition (1) of Theorem 5, we also see that the dimension of spatial variable has an impact on the stability while this is not mentioned in [25].

Remark 7. Among the three conditions of Theorem 5, condition (3) is critical and therefore we must ensure the existence of constant $\gamma>0$. Fortunately, it is not difficult to find that there must exist a constant $\gamma>0$ satisfying condition (3) if $\lambda<-h \rho$ which is easily checked. 
Theorem 8. Providing that one has the following:

(1) let $\underline{D}=\min \left\{D_{i s}: i=1, \ldots, n ; s=1, \ldots, m\right\}>0$ and denote $8 m \underline{D} / M^{2}=\chi$;

(2) $P_{i k}\left(u_{i}\left(t_{k}, x\right)\right)=-\theta_{i k} u_{i}\left(t_{k}, x\right), 1-\sqrt{1+\alpha} \leq \theta_{i k} \leq 1+$ $\sqrt{1+\alpha}, \alpha \geq 0$

(3) $\inf _{k=1,2, \ldots}\left(t_{k}-t_{k-1}\right) \geq \mu$;

(4) there exists a constant $\gamma>0$ which satisfies $\gamma+\lambda+$ $h \rho e^{\gamma \tau}>0$ and $\lambda+h \rho e^{\gamma \tau}+(\ln (1+\alpha) / \mu)<0$, where $\lambda=\max _{i=1, \ldots, n}\left(-\chi-2 a_{i}+\sum_{j=1}^{n}\left(b_{i j}^{2}+c_{i j}^{2}\right)\right)+\rho$ and $\rho=$ $n \max _{i=1, \ldots, n}\left(l_{i}^{2}\right)$;

then, the equilibrium point $u=0$ of problem (5)-(8) is globally exponentially stable with convergence rate $-(1 / 2)\left(\lambda+h \rho e^{\gamma \tau}+\right.$ $(\ln (1+\alpha) / \mu))$.

Proof. Define Lyapunov function $V$ of the form $V(t)=$ $\sum_{i=1}^{n} V_{i}(t)$, where $V_{i}(t)=\int_{\Omega} u_{i}^{2}(t, x) \mathrm{d} x$. Obviously, $V(t)$ is a piecewise continuous function with the first kind discontinuous points $t_{k}, k=1,2, \ldots$, where it is continuous from the left, that is, $V\left(t_{k}-0\right)=V\left(t_{k}\right)(k=1,2, \ldots)$. Furthermore, when $t=t_{k}(k=0,1,2, \ldots)$, it follows from condition (2) of Theorem 8 that

$$
\begin{aligned}
u_{i}^{2} & \left(t_{k}+0, x\right)-u_{i}^{2}\left(t_{k}, x\right) \\
& =\left(1-\theta_{i k}\right)^{2} u_{i}^{2}\left(t_{k}, x\right)-u_{i}^{2}\left(t_{k}, x\right) \leq \alpha u_{i}^{2}\left(t_{k}, x\right) .
\end{aligned}
$$

Thereby,

$$
V\left(t_{k}+0\right) \leq \alpha V\left(t_{k}\right)+V\left(t_{k}\right), \quad k=0,1,2, \ldots
$$

Construct another Lyapunov function $V^{*}(t)=\mathrm{e}^{\gamma\left(t-t_{0}\right)}$ $\times V(t)$, where $\gamma>0$ satisfies $\gamma+\lambda+h \rho \mathrm{e}^{\gamma \tau}>0$ and $\lambda+$ $h \rho \mathrm{e}^{\gamma \tau}+(\ln (1+\alpha) / \mu)<0$. Then, $V^{*}(t)$ is also a piecewise continuous function with the first kind discontinuous points $t_{k}, k=1,2, \ldots$, where it is continuous from the left, and for $t=t_{k}(k=0,1,2, \ldots)$, it results from (46) that

$$
V^{*}\left(t_{k}+0\right) \leq \alpha V^{*}\left(t_{k}\right)+V^{*}\left(t_{k}\right), \quad k=0,1,2, \ldots
$$

Set $t \in\left(t_{k}, t_{k+1}\right], k=0,1,2, \ldots$. Following the same procedure as in Theorem 5 , we get

$$
\begin{array}{r}
V^{*}(t) \leq V^{*}\left(t_{k}+0\right)+(\gamma+\lambda) \int_{t_{k}}^{t} V^{*}(s) \mathrm{d} s \\
+h \rho \mathrm{e}^{\gamma \tau} \sum_{j=1}^{n} \int_{t_{k}-\tau_{j}\left(t_{k}\right)}^{t-\tau_{j}(t)} \mathrm{e}^{\gamma\left(\theta-t_{0}\right)} V_{j}(\theta) \mathrm{d} \theta \\
t \in\left(t_{k}, t_{k+1}\right], \quad k=0,1,2, \ldots
\end{array}
$$

The relations (47) and (48) yield

$$
\begin{aligned}
& V^{*}(t)-V^{*}\left(t_{k}\right) \\
& \leq \alpha V^{*}\left(t_{k}\right)+(\gamma+\lambda) \int_{t_{k}}^{t} V^{*}(s) \mathrm{d} s \\
& +h \rho \mathrm{e}^{\gamma \tau} \sum_{j=1}^{n} \int_{t_{k}-\tau_{j}\left(t_{k}\right)}^{t-\tau_{j}(t)} \mathrm{e}^{\gamma\left(\theta-t_{0}\right)} V_{j}(\theta) \mathrm{d} \theta \\
& \quad t \in\left(t_{k}, t_{k+1}\right], \quad k=0,1,2, \ldots
\end{aligned}
$$

By induction argument, we reach

$$
\begin{aligned}
& V^{*}\left(t_{k}\right)-V^{*}\left(t_{k-1}\right) \\
& \leq \alpha V^{*}\left(t_{k-1}\right)+(\gamma+\lambda) \int_{t_{k-1}}^{t_{k}} V^{*}(s) \mathrm{d} s \\
& +h \rho e^{\gamma \tau} \sum_{j=1}^{n} \int_{t_{k-1}-\tau_{j}\left(t_{k-1}\right)}^{t_{k}-\tau_{j}\left(t_{k}\right)} \mathrm{e}^{\gamma\left(\theta-t_{0}\right)} V_{j}(\theta) \mathrm{d} \theta, \\
& \vdots \\
& V^{*}\left(t_{2}\right)-V^{*}\left(t_{1}\right) \\
& \leq \alpha V^{*}\left(t_{1}\right)+(\gamma+\lambda) \int_{t_{1}}^{t_{2}} V^{*}(s) \mathrm{d} s \\
& +h \rho \mathrm{e}^{\gamma \tau} \sum_{j=1}^{n} \int_{t_{1}-\tau_{j}\left(t_{1}\right)}^{t_{2}-\tau_{j}\left(t_{2}\right)} \mathrm{e}^{\gamma\left(\theta-t_{0}\right)} V_{j}(\theta) \mathrm{d} \theta, \\
& V^{*}\left(t_{1}\right)-V^{*}\left(t_{0}\right) \\
& \leq h \rho \mathrm{e}^{\gamma \tau} \sum_{j=1}^{n} \int_{t_{0}-\tau_{j}\left(t_{0}\right)}^{t_{1}-\tau_{j}\left(t_{1}\right)} \mathrm{e}^{\gamma\left(\theta-t_{0}\right)} V_{j}(\theta) \mathrm{d} \theta . \\
& \left.t_{t_{0}}\right)+(\gamma+\lambda) V^{*}(s) \mathrm{d} s
\end{aligned}
$$

Hence,

$$
\begin{aligned}
& V^{*}(t)-V^{*}\left(t_{0}\right) \\
& \leq \alpha V^{*}\left(t_{0}\right)+(\gamma+\lambda) \int_{t_{0}}^{t} V^{*}(s) \mathrm{d} s+h \rho \mathrm{e}^{\gamma \tau} \\
& \times \sum_{j=1}^{n} \int_{t_{0}-\tau_{j}\left(t_{0}\right)}^{t-\tau_{j}(t)} \mathrm{e}^{\gamma\left(\theta-t_{0}\right)} V_{j}(\theta) \mathrm{d} \theta+\alpha \sum_{t_{0}<t_{k}<t} V\left(t_{k}\right) \\
& \leq \alpha V^{*}\left(t_{0}\right)+\left(\gamma+\lambda+h \rho \mathrm{e}^{\gamma \tau}\right) \int_{t_{0}}^{t} V^{*}(s) d s \\
&+h \rho \mathrm{e}^{\gamma \tau} \sum_{j=1}^{n} \int_{t_{0}-\tau_{j}\left(t_{0}\right)}^{t_{0}} \mathrm{e}^{\gamma\left(\theta-t_{0}\right)} V_{j}(\theta) \mathrm{d} \theta+\alpha \sum_{t_{0}<t_{k}<t} V\left(t_{k}\right) \\
& t \in\left(t_{k}, t_{k+1}\right], k=0,1,2, \ldots
\end{aligned}
$$

Introducing $h \rho \mathrm{e}^{\gamma \tau} \sum_{j=1}^{n} \int_{t_{0}-\tau_{j}\left(t_{0}\right)}^{t_{0}} \mathrm{e}^{\gamma\left(\theta-t_{0}\right)} V_{j}(\theta) \mathrm{d} \theta \leq \tau h \rho \mathrm{e}^{\gamma \tau}$ $\overline{\times \| \varphi}_{\Omega}^{2}$ as shown in the proof of Theorem 5 into (51), (51) becomes

$$
\begin{aligned}
& V^{*}(t)-V^{*}\left(t_{0}\right) \\
& \quad \leq \alpha V^{*}\left(t_{0}\right)+\tau h \rho \mathrm{e}^{\gamma \tau} \overline{\| \varphi}_{\Omega}^{2}
\end{aligned}
$$




$$
\begin{array}{r}
+\left(\gamma+\lambda+h \rho \mathrm{e}^{\gamma \tau}\right) \int_{t_{0}}^{t} V^{*}(s) \mathrm{d} s+\alpha \sum_{t_{0}<t_{k}<t} V\left(t_{k}\right) \\
t \in\left(t_{k}, t_{k+1}\right], \quad k=0,1,2 \ldots
\end{array}
$$

It then results from Lemma 2 that, for $t \geq t_{0}$,

$$
\begin{aligned}
V^{*}(t) \leq & \left((\alpha+1) V^{*}\left(t_{0}\right)+\tau h \rho \mathrm{e}^{\gamma \tau}{\overline{\|\varphi\|_{\Omega}^{2}}}^{2}\right) \\
& \times \prod_{t_{0}<t_{k}<t}(1+\alpha) \exp \left(\left(\gamma+\lambda+h \rho \mathrm{e}^{\gamma \tau}\right)\left(t-t_{0}\right)\right) \\
= & \left((\alpha+1) V^{*}\left(t_{0}\right)+\tau h \rho \mathrm{e}^{\gamma \tau}{\overline{\|\varphi\|_{\Omega}^{2}}}^{2}\right) \\
& \times(1+\alpha)^{k} \exp \left(\left(\gamma+\lambda+h \rho \mathrm{e}^{\gamma \tau}\right)\left(t-t_{0}\right)\right) .
\end{aligned}
$$

On the other hand, since $\inf _{k=1,2, \ldots}\left(t_{k}-t_{k-1}\right) \geq \mu$, one has $k \leq\left(t_{k}-t_{0}\right) / \mu$. Thereby,

$$
\begin{aligned}
(1+\alpha)^{k} & \leq \exp \left\{\frac{\ln (1+\alpha)}{\mu}\left(t_{k}-t_{0}\right)\right\} \\
& \leq \exp \left\{\frac{\ln (1+\alpha)}{\mu}\left(t-t_{0}\right)\right\}
\end{aligned}
$$

and (53) can be rewritten as

$$
\begin{aligned}
V^{*}(t) \leq & \left((\alpha+1) V^{*}\left(t_{0}\right)+\tau h \rho \mathrm{e}^{\gamma \tau}{\overline{\|\varphi\|_{\Omega}^{2}}}^{2}\right. \\
& \times \exp \left(\left(\gamma+\lambda+h \rho \mathrm{e}^{\gamma \tau}+\frac{\ln (1+\alpha)}{\mu}\right)\left(t-t_{0}\right)\right)
\end{aligned}
$$

which implies

$$
\begin{aligned}
& \left\|u\left(t, x ; t_{0}, \varphi\right)\right\|_{\Omega} \\
& \leq \sqrt{\left(\alpha+1+\tau h \rho \mathrm{e}^{\gamma \tau}\right)} \overline{\|\varphi\|_{\Omega}} \\
& \quad \times \exp \left(\frac{1}{2}\left(\lambda+h \rho \mathrm{e}^{\gamma \tau}+\frac{\ln (1+\alpha)}{\mu}\right)\left(t-t_{0}\right)\right), \\
& t \geq t_{0} .
\end{aligned}
$$

The proof is completed.

As

$$
\begin{aligned}
& 2 \sum_{j=1}^{n} b_{i j} \int_{\Omega} u_{i}(t, x) f\left(u_{j}(t, x)\right) \mathrm{d} x \\
& \quad \leq \sum_{j=1}^{n} \int_{\Omega}\left(\varepsilon_{1} b_{i j}^{2} u_{i}^{2}(t, x)+\frac{l_{j}^{2}}{\varepsilon_{1}} u_{j}^{2}(t, x)\right) \mathrm{d} x, \\
& 2 \sum_{j=1}^{n} c_{i j} \int_{\Omega} u_{i}(t, x) f\left(u_{j}\left(t-\tau_{j}(t), x\right)\right) \mathrm{d} x \\
& \quad \leq \sum_{j=1}^{n} \int_{\Omega}\left(\varepsilon_{2} c_{i j}^{2} u_{i}^{2}(t, x)+\frac{l_{j}^{2}}{\varepsilon_{2}} u_{j}^{2}\left(t-\tau_{j}(t), x\right)\right) \mathrm{d} x
\end{aligned}
$$

hold for any $\varepsilon_{1}, \varepsilon_{2}>0$. In the sequel, analogous to the proofs of Theorems 5 and 8 we arrive at the following.

Theorem 9. Provided that one has the following:

(1) let $\underline{D}=\min \left\{D_{i s}: i=1, \ldots, n ; s=1, \ldots, m\right\}>0$ and denote $8 m \underline{D} / M^{2}=\chi$;

(2) $P_{i k}\left(u_{i}\left(t_{k}, x\right)\right)=-\theta_{i k} u_{i}\left(t_{k}, x\right), 0 \leq \theta_{i k} \leq 2$;

(3) there exist constants $\gamma>0$ and $\varepsilon_{1}, \varepsilon_{2}>0$ such that $\gamma+\lambda+h \rho e^{\gamma \tau}>0$ and $\lambda+h \rho e^{\gamma \tau}<0$, where $\lambda=\max _{i=1, \ldots, n}\left(-\chi-2 a_{i}+\sum_{j=1}^{n}\left(\varepsilon_{1} b_{i j}^{2}+\varepsilon_{2} c_{i j}^{2}\right)\right)+$ $\left(n / \varepsilon_{1}\right) \max _{i=1, \ldots, n}\left(l_{i}^{2}\right)$ and $\rho=\left(n / \varepsilon_{2}\right) \max _{i=1, \ldots, n}\left(l_{i}^{2}\right)$;

then, the equilibrium point $u=0$ of problem (5)-(8) is globally exponentially stable with convergence rate $-\left(\lambda+h \rho e^{\gamma \tau}\right) / 2$.

Remark 10. According to Theorem 5, we know that there must exist constant $\gamma>0$ satisfying condition (3) of Theorem 9 if there are constants $\varepsilon_{1}, \varepsilon_{2}>0$ such that $\lambda<-h \rho$.

Theorem 11. Assume that one has the following:

(1) let $\underline{D}=\min \left\{D_{i s}: i=1, \ldots, n ; s=1, \ldots, m\right\}>0$ and denote $8 m \underline{D} / M^{2}=\chi$;

(2) $P_{i k}\left(u_{i}\left(t_{k}, x\right)\right)=-\theta_{i k} u_{i}\left(t_{k}, x\right), 1-\sqrt{1+\alpha} \leq \theta_{i k} \leq 1+$ $\sqrt{1+\alpha}, \alpha \geq 0$

(3) $\inf _{k=1,2, \ldots}\left(t_{k}-t_{k-1}\right) \geq \mu$;

(4) there exist constants $\gamma>0$ and $\varepsilon_{1}, \varepsilon_{2}>0$ such that $\gamma+\lambda+h \rho e^{\gamma \tau}>0$ and $\lambda+h \rho e^{\gamma \tau}+\ln (1+\alpha) / \mu<0$, where $\lambda=\max _{i=1, \cdots, n}\left(-\chi-2 a_{i}+\sum_{j=1}^{n}\left(\varepsilon_{1} b_{i j}^{2}+\varepsilon_{2} c_{i j}^{2}\right)\right)+$ $\left(n / \varepsilon_{1}\right) \max _{i=1, \ldots, n}\left(l_{i}^{2}\right)$ and $\rho=\left(n / \varepsilon_{2}\right) \max _{i=1, \ldots, n}\left(l_{i}^{2}\right)$;

then, the equilibrium point $u=0$ of problem (5)-(8) is globally exponentially stable with convergence rate $-(1 / 2)\left(\lambda+h \rho e^{\gamma \tau}+\right.$ $(\ln (1+\alpha) / \mu))$.

Further, on the condition that $\left|P_{i k}\left(u_{i}\left(t_{k}, x\right)\right)\right| \leq \theta_{i k} \mid u_{i} \times$ $\left(t_{k}, x\right) \mid$, where $\theta_{i k}^{2} \leq(\alpha-1) / 2$ and $\alpha \geq 1$, we obtain, for $t=t_{k}(k=1,2, \ldots)$,

$$
\begin{aligned}
& u_{i}^{2}\left(t_{k}+0, x\right)-u_{i}^{2}\left(t_{k}, x\right) \\
& \quad=\left(P_{i k}\left(u_{i}\left(t_{k}, x\right)\right)+u_{i}\left(t_{k}, x\right)\right)^{2}-u_{i}^{2}\left(t_{k}, x\right) \\
& \quad \leq 2\left(u_{i}\left(t_{k}, x\right)\right)^{2}+2\left(P_{i k}\left(u_{i}\left(t_{k}, x\right)\right)\right)^{2}-u_{i}^{2}\left(t_{k}, x\right) \\
& \quad \leq\left(2+2 \theta_{i k}^{2}\right)\left(u_{i}\left(t_{k}, x\right)\right)^{2}-u_{i}^{2}\left(t_{k}, x\right) \\
& \quad \leq \alpha u_{i}^{2}\left(t_{k}, x\right) .
\end{aligned}
$$

Identical with the proof of Theorem 8, we reach the following.

Theorem 12. Assume that one has the following:

(1) let $\underline{D}=\min \left\{D_{i s}: i=1, \ldots, n ; s=1, \ldots, m\right\}>0$ and denote $8 m \underline{D} / M^{2}=\chi$;

(2) $\left|P_{i k}\left(u_{i}\left(t_{k}, x\right)\right)\right| \leq \theta_{i k}\left|u_{i}\left(t_{k}, x\right)\right|$, where $\theta_{i k}^{2} \leq(\alpha-1) / 2$ and $\alpha \geq 1$; 
(3) $\inf _{k=1,2, \ldots}\left(t_{k}-t_{k-1}\right) \geq \mu$;

(4) there exist constants $\gamma>0$ and $\varepsilon_{1}, \varepsilon_{2}>0$ such that $\gamma+\lambda+h \rho e^{\gamma \tau}>0$ and $\lambda+h \rho e^{\gamma \tau}+(\ln (1+\alpha) / \mu)<0$, where $\lambda=\max _{i=1, \ldots, n}\left(-\chi-2 a_{i}+\sum_{j=1}^{n}\left(\varepsilon_{1} b_{i j}^{2}+\varepsilon_{2} c_{i j}^{2}\right)\right)+$ $\left(n / \varepsilon_{1}\right) \max _{i=1, \ldots, n}\left(l_{i}^{2}\right)$ and $\rho=\left(n / \varepsilon_{2}\right) \max _{i=1, \ldots, n}\left(l_{i}^{2}\right)$;

then, the equilibrium point $u=0$ of problem (5)-(8) is globally exponentially stable with convergence rate $-(1 / 2)\left(\lambda+h \rho e^{\gamma \tau}+\right.$ $(\ln (1+\alpha) / \mu))$

Remark 13. Different from Theorems 5-11, the impulsive part in Theorem 12 could be nonlinear and this will be of more applicability. Actually, Theorems 5-11 can be regarded as the special cases of Theorem 12.

\section{Examples}

Example 14. Consider system (5)-(8) equipped with $P_{i k}\left(u_{i}\right.$ $\left.\left(t_{k}, x\right)\right)=1.343 u_{i}\left(t_{k}, x\right)$. Let $n=2, m=2, \Omega=[0,1.5] \times[0,2]$, $\tau_{j}(t)=(3 / 4) \arctan (t), a_{1}=a_{2}=6.5,\left(D_{i s}\right)_{2 \times 2}=\left(\begin{array}{ll}1.2 & 2.3 \\ 2.2 & 1.5\end{array}\right)$, $\left(b_{i j}\right)_{2 \times 2}=\left(\begin{array}{ll}-0.23 & 1.3 \\ -0.14 & 3.2\end{array}\right),\left(c_{i j}\right)_{2 \times 2}=\left(\begin{array}{cc}-0.1 & -0.2 \\ 0.25 & -0.13\end{array}\right)$, and $f_{j}\left(u_{j}\right)=$ $(\sqrt{2} / 4)\left(\left|u_{j}+1\right|-\left|u_{j}-1\right|\right)$.

For $M=2$ and $\underline{D}=1.2$, we compute $\chi=4.8$. This, together with $l_{i}=\sqrt{2} / 2$, yields

$$
\begin{gathered}
\rho=n \max _{i=1, \ldots, n}\left(l_{i}^{2}\right)=1 \\
\lambda=\max _{i=1, \ldots, n}\left(-\chi-2 a_{i}+\sum_{j=1}^{n}\left(b_{i j}^{2}+c_{i j}^{2}\right)\right)+\rho=-6.461 .
\end{gathered}
$$

Let $h=4$. Since $\lambda=-6.461<-4=-h \rho$, we conclude from Theorem 5 that the equilibrium point $u=0$ of this system is globally exponentially stable.

Example 15. Consider system (5)-(8) equipped with $P_{i k}\left(u_{i}\right.$ $\left.\left(t_{k}, x\right)\right)=\arctan \left(0.5 u_{i}\left(t_{k}, x\right)\right)$. Let $n=2, m=2, \tau_{j}(t)=$ $(1 / \pi) \arctan (t), \Omega=[0,1.5] \times[0,2], a_{i}=6.5,\left(D_{i s}\right)_{2 \times 2}=$ $\left(\begin{array}{ll}1.2 & 2.3 \\ 2.2 & 3.5\end{array}\right),\left(b_{i j}\right)_{2 \times 2}=\left(\begin{array}{ll}-0.23 & 1.3 \\ -0.14 & 3.2\end{array}\right),\left(c_{i j}\right)_{2 \times 2}=\left(\begin{array}{cc}-0.1 & -0.2 \\ 0.25 & -0.13\end{array}\right)$, $f_{j}\left(u_{j}\right)=(\sqrt{2} / 4)\left(\left|u_{j}+1\right|-\left|u_{j}-1\right|\right)$, and $t_{k}=t_{k-1}+2 k$.

For $M=2$ and $\underline{D}=1.2$, we compute $\chi=4.8$. This, together with $l_{i}=\sqrt{2} / 2$ and $\varepsilon_{1}=\varepsilon_{2}=1$, yields

$$
\begin{gathered}
\rho=\frac{n}{\varepsilon_{2}} \max _{i=1, \ldots, n}\left(l_{i}^{2}\right)=1, \\
\lambda=\max _{i=1, \ldots, n}\left(-\chi-2 a_{i}+\sum_{j=1}^{n}\left(\varepsilon_{1} b_{i j}^{2}+\varepsilon_{2} c_{i j}^{2}\right)\right) \\
+\frac{n}{\varepsilon_{1}} \max _{i=1, \ldots, n}\left(l_{i}^{2}\right)=-6.461 .
\end{gathered}
$$

Letting $\tau=0.5, h=4, \mu=2$, and $\alpha=1.5$, we can find $\gamma=0.78$ satisfying

$$
\begin{gathered}
\gamma+\lambda+h \rho \mathrm{e}^{\gamma \tau}=0.2269>0, \\
\lambda+h \rho \mathrm{e}^{\gamma \tau}+\frac{\ln (1+\alpha)}{\mu}=-0.0949<0 .
\end{gathered}
$$

It is then concluded from Theorem 12 that this system is globally exponentially stable.

\section{Acknowledgment}

The work is supported by National Natural Science Foundation of China under Grant nos. 60904028, 71271118, and 71171116.

\section{References}

[1] L. O. Chua and L. Yang, "Cellular neural networks: theory," IEEE Transactions on Circuits and Systems, vol. 35, no. 10, pp. 1257$1272,1988$.

[2] L. O. Chua and L. Yang, "Cellular neural networks: applications," IEEE Transactions on Circuits and Systems, vol. 35, no. 10, pp. 1273-1290, 1988.

[3] J. Cao, "New results concerning exponential stability and periodic solutions of delayed cellular neural networks," Physics Letters A, vol. 307, no. 2-3, pp. 136-147, 2003.

[4] J. Cao, "On stability of cellular neural networks with delay," IEEE Transactions on Circuits and Systems I, vol. 40, pp. 157-165, 1993.

[5] P. P. Civalleri and M. Gilli, "A set of stability criteria for delayed cellular neural networks," IEEE Transactions on Circuits and Systems. I. Fundamental Theory and Applications, vol. 48, no. 4, pp. 494-498, 2001.

[6] J. J. Hopfield, "Neurons with graded response have collective computational properties like those of two-state neurons," Proceedings of the National Academy of Sciences of the United States of America, vol. 81, pp. 3088-3092, 1984.

[7] J. Yan and J. Shen, "Impulsive stabilization of functionaldifferential equations by Lyapunov-Razumikhin functions," Nonlinear Analysis. Theory, Methods \& Applicationss, vol. 37, no. 2, pp. 245-255, 1999.

[8] X. Z. Liu and Q. Wang, "Impulsive stabilization of high-order Hopfield-type neural networks with time-varying delays," IEEE Transactions on Neural Networks, vol. 19, pp. 71-79, 2008.

[9] X. Z. Liu, "Stability results for impulsive differential systems with applications to population growth models," Dynamics and Stability of Systems, vol. 9, no. 2, pp. 163-174, 1994.

[10] S. Arik and V. Tavsanoglu, "On the global asymptotic stability of delayed cellular neural networks," IEEE Transactions on Circuits and Systems. I. Fundamental Theory and Applications, vol. 47, no. 4, pp. 571-574, 2000.

[11] L. O. Chua and T. Roska, "Stability of a class of nonreciprocal cellular neural networks," IEEE Transactions on Circuits and Systems I, vol. 37, pp. 1520-1527, 1990.

[12] Z. H. Guan and G. Chen, "On delayed impulsive Hopfield neural networks," Neural Network, vol. 12, pp. 273-280, 1999.

[13] Q. Zhang, X. Wei, and J. Xu, "On global exponential stability of delayed cellular neural networks with time-varying delays," Applied Mathematics and Computation, vol. 162, no. 2, pp. 679686, 2005.

[14] D. D. Baĭnov and P. S. Simeonov, Systems with Impulse Effect, Ellis Horwood, Chichester, UK, 1989.

[15] I. M. Stamova, Stability Analysis of Impulsive Functional Differential Equations, Walter de Gruyter, Berlin, Germany, 2009.

[16] M. A. Arbib, Brains, Machines, and Mathematics, Springer, New York, NY, USA, 1987. 
[17] S. Haykin, Neural Networks: A Comprehensive Foundation, Prentice-Hall, Englewood Cliffs, NJ, USA, 1998.

[18] H. Akça, R. Alassar, V. Covachev, Z. Covacheva, and E. Al-Zahrani, "Continuous-time additive Hopfield-type neural networks with impulses," Journal of Mathematical Analysis and Applications, vol. 290, no. 2, pp. 436-451, 2004.

[19] G. T. Stamov, "Almost periodic models of impulsive Hopfield neural networks," Journal of Mathematics of Kyoto University, vol. 49, no. 1, pp. 57-67, 2009.

[20] G. T. Stamov and I. M. Stamova, "Almost periodic solutions for impulsive neural networks with delay," Applied Mathematical Modelling, vol. 31, pp. 1263-1270, 2007.

[21] S. Ahmad and I. M. Stamova, "Global exponential stability for impulsive cellular neural networks with time-varying delays," Nonlinear Analysis. Theory, Methods \& Applications, vol. 69, no. 3, pp. 786-795, 2008.

[22] X. Liu and K. L. Teo, "Exponential stability of impulsive highorder Hopfield-type neural networks with time-varying delays," IEEE Transactions on Neural Networks, vol. 16, pp. 1329-1339, 2005.

[23] Y. Zhang and Q. Luo, "Global exponential stability of impulsive cellular neural networks with time-varying delays via fixed point theory," Advances in Difference Equations, vol. 2013, article 23, 2013.

[24] Y. Zhang and M. Zhang, "Stability analysis for impulsive reaction-diffusion Cohen-Grossberg neural networks with time-varying delays," Journal of Nanjing University of Information Science and Technology, vol. 4, no. 3, pp. 213-219, 2012.

[25] X. Zhang, S. Wu, and K. Li, "Delay-dependent exponential stability for impulsive Cohen-Grossberg neural networks with time-varying delays and reaction-diffusion terms," Communications in Nonlinear Science and Numerical Simulation, vol. 16, no. 3, pp. 1524-1532, 2011.

[26] J. Pan and S. Zhong, "Dynamical behaviors of impulsive reaction-diffusion Cohen-Grossberg neural network with delay," Neurocomputing, vol. 73, pp. 1344-1351, 2010.

[27] K. Li and Q. Song, "Exponential stability of impulsive CohenGrossberg neural networks with time-varying delays and reaction-diffusion terms," Neurocomputing, vol. 72, pp. 231-240, 2008.

[28] J. Qiu, "Exponential stability of impulsive neural networks with time-varying delays and reaction-diffusion terms," Neurocomputing, vol. 70, pp. 1102-1108, 2007.

[29] X. Wang and D. Xu, "Global exponential stability of impulsive fuzzy cellular neural networks with mixed delays and reactiondiffusion terms," Chaos, Solitons \& Fractals, vol. 42, no. 5, pp. 2713-2721, 2009.

[30] W. Zhu, "Global exponential stability of impulsive reactiondiffusion equation with variable delays," Applied Mathematics and Computation, vol. 205, no. 1, pp. 362-369, 2008.

[31] Z. Li and K. Li, "Stability analysis of impulsive Cohen-Grossberg neural networks with distributed delays and reaction-diffusion terms," Applied Mathematical Modelling, vol. 33, no. 3, pp. 13371348, 2009.

[32] Z. Li and K. Li, "Stability analysis of impulsive fuzzy cellular neural networks with distributed delays and reaction-diffusion terms," Chaos, Solitons and Fractals, vol. 42, no. 1, pp. 492-499, 2009.

[33] J. Pan, X. Liu, and S. Zhong, "Stability criteria for impulsive reaction-diffusion Cohen-Grossberg neural networks with time-varying delays," Mathematical and Computer Modelling, vol. 51, no. 9-10, pp. 1037-1050, 2010.
[34] Y. Zhang and Q. Luo, "Novel stability criteria for impulsive delayed reaction-diffusion Cohen-Grossberg neural networks via Hardy-Poincarè inequality," Chaos, Solitons \& Fractals, vol. 45, no. 8, pp. 1033-1040, 2012.

[35] Y. Zhang and Q. Luo, "Global exponential stability of impulsive delayed reaction-diffusion neural networks via Hardy-Poincarè Inequality," Neurocomputing, vol. 83, pp. 198-204, 2012.

[36] Y. Zhang, "Asymptotic stability of impulsive reaction-diffusion cellular neural networks with time-varying delays," Journal of Applied Mathematics, vol. 2012, Article ID 501891, 17 pages, 2012.

[37] V. Lakshmikantham, D. D. Bainov, and P. S. Simeonov, Theory of Impulsive Differential Equations, World Scientific, Singapore, 1989.

[38] W.-S. Cheung, "Some new Poincaré-type inequalities," Bulletin of the Australian Mathematical Society, vol. 63, no. 2, pp. 321-327, 2001. 


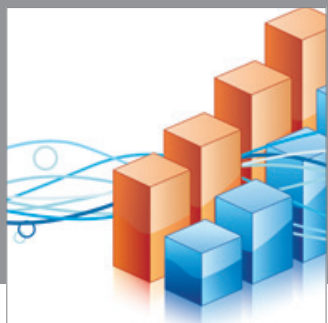

Advances in

Operations Research

mansans

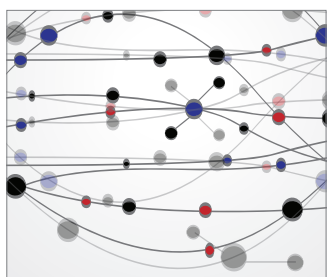

The Scientific World Journal
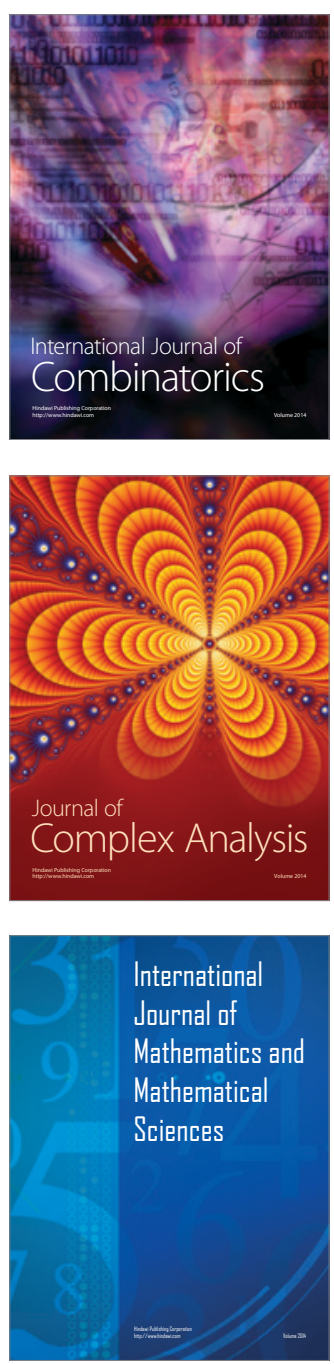
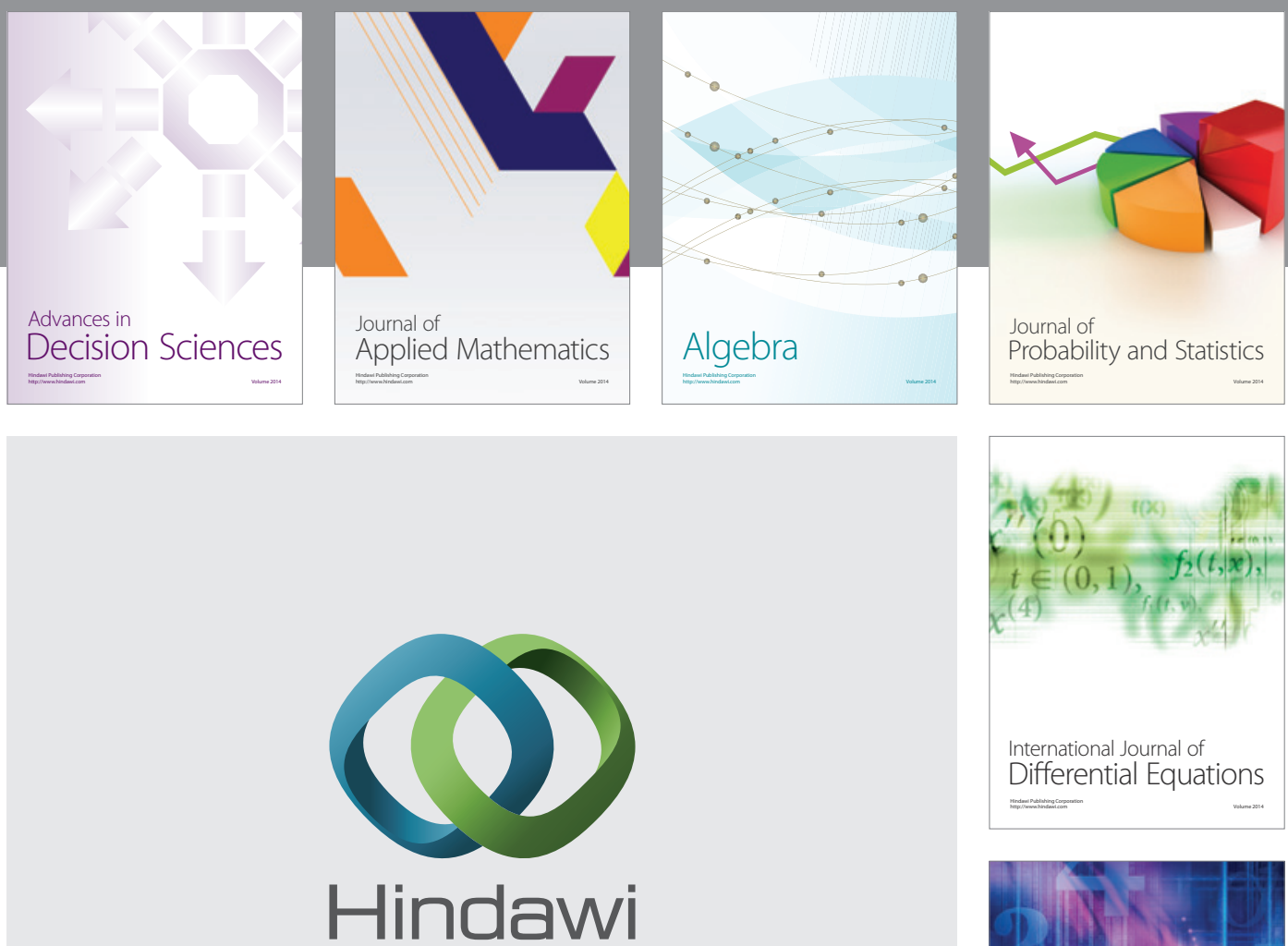

Submit your manuscripts at http://www.hindawi.com
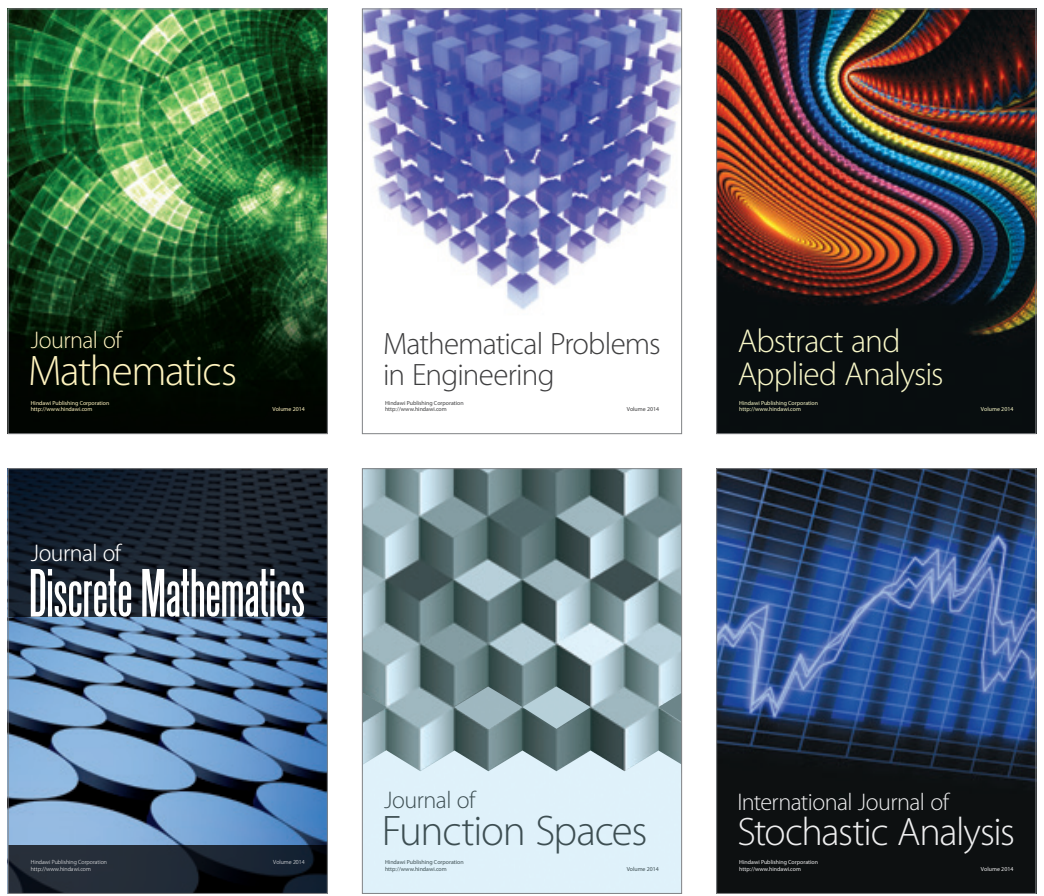

Journal of

Function Spaces

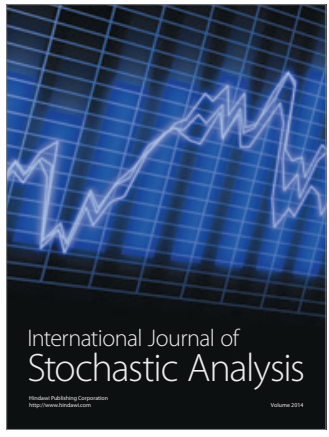

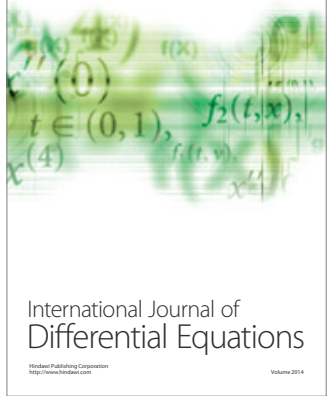
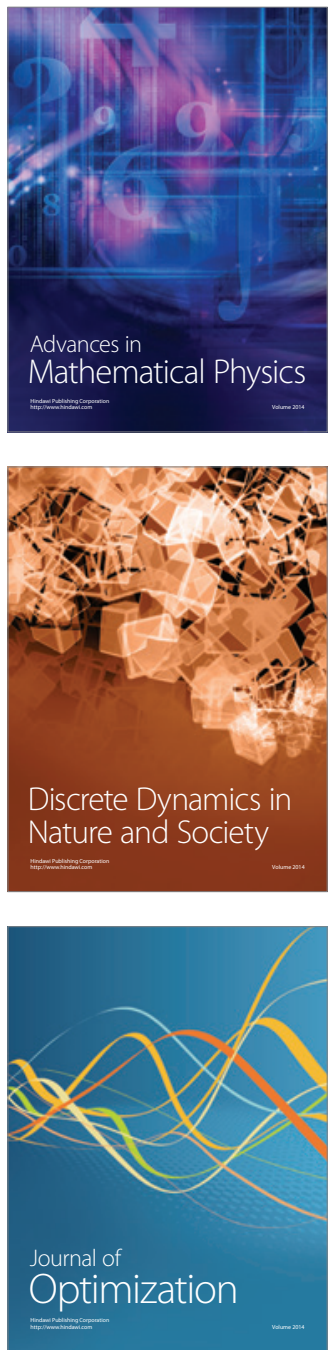